\title{
Univariate versus multivariate surrogates in the single-trial setting
}

\author{
Wim Van der Elst* \\ The Janssen Pharmaceutical companies of Johnson \& Johnson \\ Ariel Alonso Abad \\ I-BioStat, KU Leuven \\ Helena Geys \\ The Janssen Pharmaceutical companies of Johnson \& Johnson
}

\author{
Paul Meyvisch \\ Galapagos N.V. \\ Luc Bijnens
}

The Janssen Pharmaceutical companies of Johnson \& Johnson

Rudradev Sengupta

The Janssen Pharmaceutical companies of Johnson \& Johnson

and

Geert Molenberghs

I-BioStat, KU Leuven \& UHasselt

December 28, 2018

${ }^{*}$ The research leading to the results described in this paper has received funding from the European Seventh Framework programme [FP7 2007 - 2013] under grant agreement Nr. 602552. 


\begin{abstract}
In spite of medical and methodological advances, the identification of good surrogate endpoints has remained a challenging endeavour. This may, at least partially, be attributable to the fact that most researchers have only focused on univariate surrogates endpoints. In the present work, we argue in favour of using multivariate surrogates and introduce two new complementary metrics to assess their validity. The first one, the so-called Individual Causal Association, quantifies the association between the individual causal treatment effects on the multivariate surrogate and true endpoints, while the second one quantifies the treatment-corrected association between the multivariate surrogate and the true endpoint outcomes. The newly proposed methodology is implemented in the R package Surrogate and a Web Appendix, detailing how the analysis can be conducted in practice, is provided.
\end{abstract}

Keywords: Causal inference; information theory; multivariate surrogate endpoints 


\section{Introduction}

The duration, complexity and cost of a clinical trial are substantially affected by the endpoints that are used to assess treatment efficacy (Burzykowski et al., 2005). In some situations, the most credible indicator of therapeutic response, the so-called true endpoint, may be distant in time (e.g., survival time in early cancer stages), rare (e.g., pregnancy in severe luteinizing hormone deficiency), ethically challenging (e.g., procedures that involve a non-negligible health risk), or expensive (e.g., imaging data). An appealing strategy in these circumstances is to substitute the true endpoint by a 'replacement endpoint' that can be measured earlier, occurs more frequently, is more ethically acceptable, and/or is cheaper. If such a replacement endpoint allows for the accurate prediction of the treatment effect on the true endpoint, it is termed a surrogate endpoint (Buyse and Molenberghs, 1998; Buyse et al., 2000; Freedman, Graubard and Schatzkin, 1992; Prentice, 1989).

In spite of important methodological advances, the identification of good surrogate endpoints remains challenging (Alonso et al., 2016; Buyse et al., 2000, 2010). In fact, as practice has shown, the evaluation of surrogate endpoints is often a strenuous process with respect to both the initial demonstration of a relationship between a putative surrogate and the true endpoint, and its subsequent statistical validation (Buyse et al., 2010). This may, at least partially, be attributable to the fact that most researchers have only focused on univariate surrogate endpoints. Indeed, it has often implicitly been assumed that the treatment effect on the true endpoint can be accurately predicted based on the treatment effect on a single surrogate endpoint. However, given the complex nature of many diseases and the various therapeutic pathways in which a treatment can impact the true endpoint, this assumption may be overly optimistic. In the present work, it is argued that the use of multivariate surrogate endpoints may allow for a better prediction of the treatment effect on

the true endpoint. A new evaluation strategy for multivariate surrogates is proposed within a causal-inference framework. The methodology uses information-theoretic concepts, and builds upon previous work in this area (Alonso et al., 2015; Van der Elst, Molenberghs and Alonso, 2016).

The remainder of this paper is organised as follows. In Section 2, Rubin's causal inference model (Rubin, 1986) is introduced. In Sections 3 and 4, the multivariate individual 
causal association is defined and the identifiability issues that are encountered in estimating this metric are discussed. In Section 5, a complementary metric of surrogacy is introduced to assess the association between the multivariate surrogate and the true endpoint. In Section 6, the newly developed methodology is exemplified in a case study (the transPAT microbiome study). Finally, some critical comments regarding the newly proposed methodology are given in Section 8.

A problem often faced by applied statisticians and researchers when evaluating surrogate endpoints is the lack of user-friendly software to conduct the complex analyses. To address this issue, the multivariate surrogate evaluation methodology, proposed in the current paper, has been incorporated into $\mathrm{R}$ package Surrogate (available for download at CRAN). In a Web appendix that accompanies this paper, it is shown how the package can be used to conduct the surrogacy analyses in practice.

\section{Causal-inference model}

In the remainder of the paper, it will be assumed that data on a univariate true endpoint $T$ and a multivariate surrogate endpoint $\boldsymbol{S}=\left(S_{1}, S_{2}, \ldots, S_{p}\right)$ were collected for $N$ patients in a single clinical trial. Note that no sub-index for patients is used to simplify the notation. It will also be assumed that only two treatments are under evaluation $(Z=0 / 1)$ in a parallel study design.

Rubin's model for causal inference (Rubin, 1986) assumes that each patient has two potential outcomes for $T$ : an outcome $T_{0}$ that would be observed under the control treatment $(Z=0)$, and an outcome $T_{1}$ that would be observed under the experimental treatment $(Z=1)$. Using evident notation, let us now consider the $2(p+1)$ dimensional vector of potential outcomes $\boldsymbol{Y}=\left(T_{0}, T_{1}, S_{10}, S_{11}, S_{20}, S_{21}, \ldots, S_{p 0}, S_{p 1}\right)^{\prime}$ and the corresponding vector of individual causal treatment effects $\boldsymbol{\Delta}=\left(\Delta T, \boldsymbol{\Delta} \boldsymbol{S}^{\prime}\right)^{\prime}$, where $\Delta T=T_{1}-T_{0}$ and $\Delta \boldsymbol{S}=\left(\Delta S_{1}, \Delta S_{2}, \ldots \ldots, \Delta S_{p}\right)^{\prime}$ with $\Delta S_{k}=S_{k 1}-S_{k 0}$. The so-called fundamental problem of causal inference states that only one of the potential outcomes associated with the true and surrogate endpoints are observed in practice. Therefore, $\boldsymbol{\Delta}$ cannot be estimated from the data (Holland, 1986).

Based on $\boldsymbol{\Delta}$, one can define the expected or average causal treatment effects in the 
population of interest as $E(\boldsymbol{\Delta})=(\beta, \boldsymbol{\alpha})$, where $\beta=E(\Delta T)$ and $\boldsymbol{\alpha}=\left(\alpha_{k}\right)$ with $\alpha_{k}=$ $E\left(\Delta S_{k}\right)$. Rosenbaum and Rubin (1983) provided three identifiability conditions under which it is possible to obtain consistent estimators of the expected causal treatment effects. If $Y$ denotes the response of interest and $Y_{z}$ the potential outcome associated with $Z=z$ then the three identifiability conditions are: 1) Consistency: If $Z=z$ for a given subject then $Y_{z}=Y$ for that subject, 2) Conditional exchangeability: There is no unmeasured confounding given the data on baseline covariates $L$, i.e., $Y_{z} \perp Z \mid L=l$ for each possible value $z$ of $Z$ and $l$ of $L$ and 3) Positivity: If $f_{L}(l) \neq 0$ then $f_{Z \mid L}(z \mid l)>0$ (Fitzmaurice et al., 2009). It can easily be shown that in randomized clinical trials all conditions hold and the expected causal treatment effects can be estimated as $\beta=E(T \mid Z=1)-E(T \mid Z=0)$ and $\alpha_{k}=E\left(S_{k} \mid Z=1\right)-E\left(S_{k} \mid Z=0\right)$, where the conditional expectations are estimated using the observed means in the control and treated groups. The methodology proposed in the following sections is based only on the individual causal treatment effects and it is valid if consistency holds, i.e., it could also be applied to observational data.

Although less relevant for the estimation of the expected causal treatment effects, the distribution of the vector of potential outcomes plays an important role in the surrogate evaluation context. In the following, it will be further assumed that $\boldsymbol{Y} \sim N(\boldsymbol{\mu}, \boldsymbol{\Sigma})$, where $\boldsymbol{\mu}=\left(\mu_{T_{0}}, \mu_{T_{1}}, \mu_{S_{10}}, \mu_{S_{11}}, \mu_{S_{20}}, \mu_{S_{21}}, \ldots, \mu_{S_{p 0}}, \mu_{S_{p 1}}\right)^{\prime}$ and

$$
\boldsymbol{\Sigma}=\left(\begin{array}{ccccccccc}
\sigma_{T_{0} T_{0}} & \sigma_{T_{0} T_{1}} & \sigma_{T_{0} S_{10}} & \sigma_{T_{0} S_{11}} & \sigma_{T_{0} S_{20}} & \sigma_{T_{0} S_{21}} & \cdots & \sigma_{T_{0} S_{p 0}} & \sigma_{T_{0} S_{p 1}} \\
\sigma_{T_{0} T_{1}} & \sigma_{T_{1} T_{1}} & \sigma_{T_{1} S_{10}} & \sigma_{T_{1} S_{11}} & \sigma_{T_{1} S_{20}} & \sigma_{T_{1} S_{21}} & \cdots & \sigma_{T_{1} S_{p 0}} & \sigma_{T_{1} S_{p 1}} \\
\sigma_{T_{0} S_{10}} & \sigma_{T_{1} S_{11}} & \sigma_{S_{10} S_{10}} & \sigma_{S_{10} S_{11}} & \sigma_{S_{10} S_{20}} & \sigma_{S_{10} S_{21}} & \cdots & \sigma_{S_{10} S_{p 0}} & \sigma_{S_{10} S_{p 1}} \\
\sigma_{T_{0} S_{11}} & \sigma_{T_{1} S_{11}} & \sigma_{S_{10} S_{11}} & \sigma_{S_{11} S_{11}} & \sigma_{S_{11} S_{20}} & \sigma_{S_{11} S_{21}} & \cdots & \sigma_{S_{11} S_{p 0}} & \sigma_{S_{11} S_{p 1}} \\
\sigma_{T_{0} S_{20}} & \sigma_{T_{1} S_{20}} & \sigma_{S_{10} S_{20}} & \sigma_{S_{11} S_{20}} & \sigma_{S_{20} S_{20}} & \sigma_{S_{20} S_{21}} & \cdots & \sigma_{S_{20} S_{p 0}} & \sigma_{S_{20} S_{p 1}} \\
\sigma_{T_{0} S_{21}} & \sigma_{T_{1} S_{21}} & \sigma_{S_{20} S_{21}} & \sigma_{S_{11} S_{21}} & \sigma_{S_{20} S_{21}} & \sigma_{S_{21} S_{21}} & \cdots & \sigma_{S_{21} S_{p 0}} & \sigma_{S_{21} S_{p 1}} \\
\vdots & \vdots & \vdots & \vdots & \vdots & \vdots & \ddots & \vdots & \vdots \\
\sigma_{T_{0} S_{p 0}} & \sigma_{T_{1} S_{p 0}} & \sigma_{S_{10} S_{p 0}} & \sigma_{S_{11} S_{p 0}} & \sigma_{S_{20} S_{p 0}} & \sigma_{S_{21} S_{p 0}} & \cdots & \sigma_{S_{p 0} S_{p 0}} & \sigma_{S_{p 0} S_{p 1}} \\
\sigma_{T_{0} S_{p 1}} & \sigma_{T_{1} S_{p 1}} & \sigma_{S_{10} S_{p 1}} & \sigma_{S_{11} S_{p 1}} & \sigma_{S_{20} S_{p 1}} & \sigma_{S_{21} S_{p 1}} & \cdots & \sigma_{S_{p 0} S_{p 1}} & \sigma_{S_{p 1} S_{p 1}}
\end{array}\right) .
$$

Other authors have already used the normal distribution to build up causal-inference models in this context. For instance, Conlon, Taylor, and Elliott (2013) developed a Bayesian 
estimation strategy for the evaluation of surrogate endpoints, assuming a multivariate normal distribution for the vector of potential outcomes. Similarly, Alonso et al. (2015) and Van der Elst, Molenberghs and Alonso (2016) studied the relationship between the causal-inference and meta-analytic paradigms using normal potential outcomes as well.

Given the aforementioned distributional assumptions, it follows that $\Delta \sim N\left(\boldsymbol{\mu}_{\Delta}, \boldsymbol{\Sigma}_{\Delta}\right)$ with $\boldsymbol{\mu}_{\Delta}=(\beta, \boldsymbol{\alpha})^{\prime}$ and

$$
\boldsymbol{\Sigma}_{\boldsymbol{\Delta}}=\left(\begin{array}{cc}
\sigma_{\Delta T} & \boldsymbol{\Sigma}_{\boldsymbol{\Delta} \boldsymbol{S} \Delta T}^{\prime} \\
\boldsymbol{\Sigma}_{\boldsymbol{\Delta} \boldsymbol{S} \Delta T} & \boldsymbol{\Sigma}_{\boldsymbol{\Delta} \boldsymbol{S}}
\end{array}\right)
$$

where $\sigma_{\Delta T}=\sigma_{T_{0} T_{0}}+\sigma_{T_{1} T_{1}}-2 \rho_{T_{0} T_{1}} \sqrt{\sigma_{T_{0} T_{0}}+\sigma_{T_{1} T_{1}}}$ is the variance of $\Delta T, \boldsymbol{\Sigma}_{\boldsymbol{\Delta} \boldsymbol{S} \Delta T}$ is a $(p \times 1)$ column vector of covariances between $\Delta T$ and $\Delta S_{1}, \Delta S_{2}, \ldots, \Delta S_{p}$

$$
\Sigma_{\Delta S \Delta T}=\left(\begin{array}{c}
\sigma_{\Delta S_{1} \Delta T} \\
\sigma_{\Delta S_{2} \Delta T} \\
\vdots \\
\sigma_{\Delta S_{p} \Delta T}
\end{array}\right)
$$

and $\boldsymbol{\Sigma}_{\boldsymbol{\Delta} \boldsymbol{S}}$ is the $(p \times p)$ variance-covariance matrix of $\boldsymbol{\Delta} \boldsymbol{S}$

$$
\Sigma_{\Delta S}=\left(\begin{array}{cccc}
\sigma_{\Delta S_{1} \Delta S_{1}} & \sigma_{\Delta S_{1} \Delta S_{2}} & \cdots & \sigma_{\Delta S_{1} \Delta S_{k}} \\
\sigma_{\Delta S_{1} \Delta S_{2}} & \sigma_{\Delta S_{2} \Delta S_{2}} & \cdots & \sigma_{\Delta S_{2} \Delta S_{k}} \\
\vdots & \vdots & \ddots & \vdots \\
\sigma_{\Delta S_{1} \Delta S_{k}} & \sigma_{\Delta S_{2} \Delta S_{k}} & \cdots & \sigma_{\Delta S_{k} \Delta S_{k}}
\end{array}\right) .
$$

\section{Multivariate individual causal association}

It has been argued that understanding the association between the individual causal treatment effects on the true and surrogate endpoints is critical to assess the quality of a surrogate (Elliott, Li and Taylor, 2013). Along these lines, in the univariate setting $(p=1)$, Alonso et al. (2015) proposed to assess surrogacy using the so-called individual causal association (ICA). When both endpoints are continuous and normally distributed variables, these authors quantified the ICA using the Pearson correlation coefficient, i.e., $\rho_{\Delta}=\operatorname{corr}(\Delta T, \Delta S)$. The use of $\rho_{\Delta}$ can further be justified from an information-theoretic 
perspective. Indeed, Alonso et al. (2015) stated that $S$ is a good surrogate for $T$ if and only if $\Delta S$ conveys a substantial amount of information about $\Delta T$. The amount of uncertainty in $\Delta T$ that is expected to be removed when the value of $\Delta S$ becomes known is given by the so-called mutual information $I(\Delta T, \Delta S)$. In the univariate normal-normal setting (i.e., both $S$ and $T$ are normally distributed endpoints), there is a one-to-one relationship between $\rho_{\Delta}$ and $I(\Delta T, \Delta S)$ and thus both concepts are equivalent.

In the multivariate case, under the previous distributional assumptions, the mutual information between $\Delta T$ and $\boldsymbol{\Delta} \boldsymbol{S}$ is given by

$$
I(\Delta T, \boldsymbol{\Delta} \boldsymbol{S})=-\frac{1}{2} \log \left(\frac{\left|\boldsymbol{\Sigma}_{\boldsymbol{\Delta}}\right|}{\left|\sigma_{\Delta T}\right|\left|\boldsymbol{\Sigma}_{\boldsymbol{\Delta} \boldsymbol{S}}\right|}\right)
$$

where $|\mathbf{A}|$ denotes the determinant of matrix $\mathbf{A}$. Although it seems sensible to use this measure to quantitatively assess the appropriateness of $\boldsymbol{\Delta} \boldsymbol{S}$ to predict $\Delta T$, the absence of an upper bound for $I(\Delta T, \boldsymbol{\Delta S})$ adversely affects its interpretation. To solve this problem, we propose the use of a normalized version of the mutual information, the so-called squared informational coefficient of correlation (SICC) (Joe, 1989; Linfoot, 1957)

$$
R_{H}^{2}=1-e^{-2 I(\Delta T, \boldsymbol{\Delta} \boldsymbol{S})}=1-\frac{\left|\boldsymbol{\Sigma}_{\boldsymbol{\Delta}}\right|}{\left|\sigma_{\Delta T}\right|\left|\boldsymbol{\Sigma}_{\boldsymbol{\Delta} \boldsymbol{S}}\right|} .
$$

The SICC is always in the unit interval $[0,1]$, is invariant under bijective transformations, and takes the value zero if and only if $\Delta T$ and $\boldsymbol{\Delta} \boldsymbol{S}$ are independent. Moreover, mutual information approaches infinity when the distribution of $(\Delta T, \boldsymbol{\Delta} \boldsymbol{S})$ approaches a singular distribution, i.e., $R_{H}^{2} \approx 1$ if and only if there exists an approximate deterministic relationship between $\Delta T$ and $\boldsymbol{\Delta} \boldsymbol{S}$ (Joe, 1989). In the following, we will refer to (3) as the multivariate ICA. Taking into account that $\left|\boldsymbol{\Sigma}_{\boldsymbol{\Delta}}\right|=\left|\boldsymbol{\Sigma}_{\boldsymbol{\Delta} \boldsymbol{S}}\right|\left|\sigma_{\Delta T \mid \boldsymbol{\Delta} \boldsymbol{S}}\right|$ where $\sigma_{\Delta T \mid \boldsymbol{\Delta} \boldsymbol{S}}=\sigma_{\Delta T}-\boldsymbol{\Sigma}_{\boldsymbol{\Delta} \boldsymbol{S} \Delta T}^{\prime} \boldsymbol{\Sigma}_{\boldsymbol{\Delta} \boldsymbol{S}}^{-1} \boldsymbol{\Sigma}_{\boldsymbol{\Delta} \boldsymbol{S} \Delta T}$, one gets

$$
R_{H}^{2}=\frac{\Sigma_{\Delta S \Delta T}^{\prime} \Sigma_{\Delta S}^{-1} \Sigma_{\Delta S \Delta T}}{\sigma_{\Delta T}} .
$$

Notice also that $\sigma_{\Delta T \mid \Delta S}=\sigma_{\Delta T}\left(1-R_{H}^{2}\right)$. The following lemma summarizes some properties of $R_{H}^{2}$ that further justifies its use as a surrogate evaluation metric.

Lemma 1 Let $\boldsymbol{\Delta}=\left(\Delta T, \boldsymbol{\Delta} \boldsymbol{S}^{\prime}\right)^{\prime}$ denote the vector containing the individual causal treatment effects on $T$ and $\boldsymbol{S}$ with $\boldsymbol{\Delta} \sim N\left(\boldsymbol{\mu}_{\Delta}, \boldsymbol{\Sigma}_{\Delta}\right)$ and $\boldsymbol{\Sigma}_{\Delta}$ as given in (2). The coefficient $R_{H}^{2}$ satisfies the following properties: 
1. $R_{H}^{2}$ is invariant by bijective transformations of $\Delta T$ and $\Delta \boldsymbol{S}$

2. $0 \leq R_{H}^{2} \leq 1$

3. $R_{H}^{2}=0$ if and only if $\sigma_{\Delta S_{k} \Delta T}=0$ for all $k=1,2, \ldots, p$

4. $R_{H}^{2}=1$ if and only if there exists a deterministic relationship between $\Delta T$ and $\Delta \boldsymbol{S}$

It can easily be shown that $R_{H}^{2}$ is the maximum squared correlation between $\Delta T$ and a linear combination of $\boldsymbol{\Delta} \boldsymbol{S}$, i.e., $R_{H}^{2}=\max _{\mathbf{t}}\left[\operatorname{corr}\left(\Delta T, \boldsymbol{t}^{\prime} \boldsymbol{\Delta} \boldsymbol{S}\right)\right]^{2}$. Statement (3) in Lemma 1 shows that the multivariate ICA is zero if and only if all the individual components of $\boldsymbol{S}$ produce an ICA equal to zero as well, i.e., $R_{H}^{2}=0$ if and only if $\rho_{\Delta k}=\operatorname{corr}\left(\Delta T, \Delta S_{k}\right)=0$ for all $k$. In such a situation, $\boldsymbol{\Delta} \boldsymbol{S}$ carries no information with respect to the individual causal treatment effect on $T$ and, hence, knowing what the treatment does to the surrogates conveys no information regarding its effect on the true endpoint. On the other hand, when the multivariate ICA is close to one, then $\Delta T$ and $\boldsymbol{\Delta} \boldsymbol{S}$ are almost deterministically related and thus the treatment effect on $T$ can almost perfectly be predicted by $\boldsymbol{\Delta} \boldsymbol{S}$. A proof of the lemma can be found in the Web Appendix (Part I). Furthermore, the following lemma shows that there are important reasons to explore the use of multivariate surrogate endpoints.

Lemma 2 Let $\boldsymbol{S}_{*}=\left(\boldsymbol{S}^{\prime}, S_{*}\right)^{\prime}$ be a new $(p+1)$ dimensional surrogate endpoint, constructed by adding a new univariate surrogate $S_{*}$ to $\boldsymbol{S}$. Using obvious notation one has that $R_{H}^{2} \leq$ $R_{H *}^{2}$.

Essentially, Lemma 2 states that considering additional surrogate endpoints can only improve our capacity to predict the individual causal treatment effect on the true endpoint. This can intuitively be easily understood, as $R_{H}^{2}$ is the coefficient of multiple determination of a regression model in which $\Delta T$ is regressed on $\Delta \boldsymbol{S}$. The sample coefficient of determination is a positively biased estimate of the population coefficient of determination (Cohen et al., 2003), and the bias is larger when the dimension of $\boldsymbol{\Delta} \boldsymbol{S}$ increases. Several proposals have been made to correct for this bias (Yin and Fan, 2001). For example, the use of Wherry's equation would lead to the correction $1-\left(1-R_{H}^{2}\right) \frac{N-1}{N-p-1}$. The latter estimate (referred to as the bias-corrected $R_{H}^{2}$ ) will necessarily (and appropriately) be smaller than $R_{H}^{2}$, and the extent to which the bias-corrected $R_{H}^{2}$ is reduced will depend on 
the number of surrogates that are considered. The latter correction is also implemented in the Surrogate package.

\section{$4 \quad$ Estimating $R_{H}^{2}$}

As previously stated, due to the fundamental problem of causal inference, neither $\Delta T$ nor $\boldsymbol{\Delta} \boldsymbol{S}$ can be estimated from the data and, consequently, $R_{H}^{2}$ is not identifiable. In the univariate setting, Alonso et al. (2015) argued that there are two main strategies to deal with these identifiability issues. First, one can try to define identifiability conditions based on biological knowledge. However, this approach suffers from two important drawbacks: (i) these biological assumptions are often empirically untestable, and (ii) they often have to be supplemented with additional assumptions for which no such subject-specific knowledge exist. Different identifiability conditions may also lead to substantially different estimates of $R_{H}^{2}$, and thus to qualitatively different conclusions regarding the appropriateness of the putative surrogate(s).

A second approach is to implement a simulation-based sensitivity analysis in which $R_{H}^{2}$ is estimated across a set of plausible values for the unidentifiable parameters in $\boldsymbol{\Sigma}$. In the univariate setting, there are only four non-identifiable correlations involved in the computation of ICA (i.e., $\rho_{T_{1} S_{0}}, \rho_{T_{0} S_{1}}, \rho_{T_{0} T_{1}}$ and $\rho_{S_{0} S_{1}}$ ). Alonso et al. (2015) proposed an algorithm in which grids of values $G=\left\{g_{1}, g_{2}, \ldots, g_{k}\right\}$ are specified for the four unidentified correlations. Next, several covariance matrices $\boldsymbol{\Sigma}$ are generated using all the combinations that emanate from the specified grids, where the identifiable parameters are fixed at their estimated values. From all the previous matrices, only those that are positive definite are retained. Next, $\rho_{\Delta}$ is computed based on these positive definite matrices. In essence, the soobtained vector $\rho_{\Delta}$ quantifies the univariate ICA across all plausible 'realities', i.e., across all those scenarios where the assumptions that are made for the unidentified correlations are compatible with the observed data. The general behavior of $\rho_{\Delta}$ can subsequently be examined, e.g., by quantifying the variability and the range of its estimates. In this way, the sensitivity of the results with respect to the unverifiable assumptions (uncertainty) can be evaluated.

Note that the two previous strategies to deal with the identifiability issues are not 
mutually exclusive. In fact, the simulation-based approach allows for a straightforward incorporation of subject-specific knowledge in case it is available. For example, when it is biologically-sound to assume that the unidentified correlations are positive, then a grid $G$ with only positive values can be used when carrying out the sensitivity analysis.

In the multivariate setting, the grid-based approach to estimate the ICA becomes computationally challenging. Indeed, when multivariate surrogates are considered, the number of combinations that emanate from the specified grids for the unidentified correlations becomes extremely large rapidly. For example, when $\Delta S$ has dimension three, use of the grid $G=\{-1,-0.90, \ldots, 1\}$ for each of the 16 unidentifiable correlations would lead to a total of $21^{16}$ matrices that need to be evaluated by the algorithm.

An alternative approach can be constructed as follows. First, one specifies a (discrete or continuous) distribution for the unidentified correlations, e.g., $G \sim \operatorname{unif}(-1,1)$. Next, several covariance matrices $\boldsymbol{\Sigma}$ are generated. To this end, the unidentifiable correlations are randomly and independently sampled from $G$ and, under the consistency assumption, the identifiable parameters can be estimated using the data from the control and treated groups. In the subsequent steps the identifiable parameters are fixed at their estimated values. From the previous matrices only those that are positive definite are retained to calculate the multivariate ICA. The general behavior of $R_{H}^{2}$ can subsequently be examined in the same way as is done with $\rho_{\Delta}$ in the single-surrogate setting.

An important issue that affects the previous approach is that the probability of obtaining a positive definite matrix is low when the dimensionality of $\boldsymbol{\Sigma}$ is high (Rousseeuw and Molenberghs, 1994). To increase the efficiency of the algorithm, one can gradually buildup $\Sigma$ rather than generate it 'as a whole'. Taking into account the Sylvester's criterion, i.e., the fact that a $(p \times p)$ matrix is positive definite if and only if all its principal minors are positive definite, one can start with the upper-left $(2 \times 2)$ submatrix and randomly sample a value from $G$ for the unidentified correlation $\rho_{T_{0} T_{0}}$. When the determinant of the 'completed' $(2 \times 2)$ submatrix is positive (which will always be the case for a $(2 \times 2)$ matrix), the algorithm continues with the upper-left $(3 \times 3)$ submatrix. Here, a value for $\rho_{T_{1} S 1_{0}}$ is randomly sampled from $G$ and $\rho_{T_{0} S 1_{0}}$ is fixed at its estimated value. When the determinant of the 'completed' $(3 \times 3)$ submatrix is not positive, a new value for $\rho_{T_{1} S 1_{0}}$ 
is randomly sampled from $G$ and this procedure is repeated until a positive determinant is obtained. The algorithm continues in the same way until the full $(p \times p)$ matrix is obtained. This approach guarantees that the full $(p \times p)$ matrix will be positive definite. The methodology is computationally feasible to generate positive definite matrices of a dimension up to $(10 \times 10)$ (which corresponds to a scenario where four surrogates are considered), but it breaks down for larger matrices (see Discussion). The latter approach of gradually building up $\Sigma$ is also used in the Surrogate R package.

\section{The multivariate adjusted association}

In the univariate setting, Buyse and Molenberghs (1998) defined the adjusted association as $\gamma=\operatorname{corr}(T, S \mid Z)$. The adjusted association is not based on causal treatment effects, but is rather defined as the association between the surrogate and true endpoints, after adjusting for treatment. Although it has now been clearly established that measures of association between the surrogate and true endpoints have limited value as metrics of surrogacy, the existence of such an association is widely considered a necessary condition for the validity of a putative surrogate (Alonso et al., 2016; Fleming and DeMets, 1996). In the same spirit as was done with ICA, the univariate adjusted association can be generalized to a multivariate setting. To this end, consider the following linear regression models:

$$
\left\{\begin{array}{c}
T=\mu_{T}+\beta Z+\varepsilon_{T}, \\
S_{1}=\mu_{S_{1}}+\alpha_{1} Z+\varepsilon_{S_{1}}, \\
S_{2}=\mu_{S_{2}}+\alpha_{2} Z+\varepsilon_{S_{2}}, \\
\vdots \\
S_{p}=\mu_{S_{p}}+\alpha_{p} Z+\varepsilon_{S_{p}} .
\end{array}\right.
$$

The $\beta, \alpha_{1}, \alpha_{2}, \ldots, \alpha_{p}$ components are the expected causal treatment effects that were defined in Section 3. Further, $\left(\varepsilon_{T}, \varepsilon_{S_{1}}, \varepsilon_{S_{2}}, \ldots \varepsilon_{S_{p}}\right) \sim N\left(\mathbf{0}, \boldsymbol{\Sigma}_{\gamma}\right)$, with

$$
\boldsymbol{\Sigma}_{\gamma}=\left(\begin{array}{cc}
\sigma_{T} & \Sigma_{\boldsymbol{S} T} \\
\Sigma_{\boldsymbol{S} T}^{\prime} & \Sigma_{\boldsymbol{S S}}
\end{array}\right)
$$


The multivariate adjusted association $\gamma_{\Delta}^{2}$ can then be defined as the coefficient of determination that is obtained when the treatment-corrected $T$ (i.e., $\varepsilon_{T}$ ) is regressed on the treatment-corrected $S_{1}, S_{2}, \ldots$, and $S_{p}$ (i.e., $\varepsilon_{S_{1}}, \varepsilon_{S_{2}}, \ldots$, and $\varepsilon_{S_{p}}$, respectively)

$$
\gamma_{\Delta}^{2}=\frac{\Sigma_{S T}^{\prime} \Sigma_{S S}^{-1} \Sigma_{S T}}{\sigma_{T}} .
$$

Again, Wherry's equation can be used to correct for the positive bias of the estimate.

\section{Case study: the transPAT microbiome intervention study}

The dataset Data from the transPAT experiment (Ruiz et al., 2017) are used to illustrate the methodology. TransPAT is an animal study that was conducted to evaluate the influence of an antibiotic treatment on the immune system (Immunoglobulin A level, IgA level) and on the microbiome of an animal. The microbiome is composed of a wide variety of operational taxonomic units (OTUs), which are essentially proxies for microbial species. The transPAT dataset contains information from $N=15$ germ-free mice that received cecal contents of a donor mouse. The cecal contents of the donor mouse was either exposed (experimental treatment) or not exposed (control treatment) to a tylosin pulse. A total of $n=7$ and $n=8$ mice received the experimental and the control treatments, respectively. The relative abundance of a total of 67 OTUs was assessed at day 12 of the experiment. One of the objectives of the study is to evaluate whether the treatment effect on the OTUs conveys information on the potential treatment effect on the immune response, i.e., one wants to evaluate whether the individual causal treatment effect on the relative abundance of one or more of the 67 OTUs at day 12 of the experiment $\left(\Delta S_{1}, \Delta S_{2}\right.$, $\left.\ldots, \Delta S_{67}\right)$ is predictive for the individual causal treatment effect on IgA level at day 20 of the experiment $(\Delta T)$. Such an analysis may help understand the causal mechanism by which antibiotics affect the immune response and shed light on the interconnection between the microbiome and the immune system. The data are available on github (see https://github.com/blaser-lab/Paper-Ruiz-2017). 
Table 1: TransPAT trial. Correlations between the true endpoint $(T)$ and the surrogate endpoints $\left(S_{1}, S_{2}\right.$ and $\left.S_{2}\right)$ in the two treatment conditions.

\begin{tabular}{ccccccccc} 
& $T_{0}$ & $T_{1}$ & $S_{10}$ & $S_{12}$ & $S_{20}$ & $S_{21}$ & $S_{30}$ & $S_{31}$ \\
\hline$T_{0}$ & 1.000 & & & & & & & \\
$T_{1}$ & - & 1.000 & & & & & & \\
$S_{10}$ & 0.893 & - & 1.000 & & & & & \\
$S_{11}$ & - & -0.156 & - & 1.000 & & & & \\
$S_{20}$ & -0.746 & - & -0.535 & - & 1.000 & & & \\
$S_{21}$ & - & -0.639 & - & 0.571 & - & 1.000 & & \\
$S_{30}$ & 0.038 & - & -0.331 & - & -0.208 & - & 1.000 & \\
$S_{31}$ & - & -0.564 & - & 0.636 & - & 0.972 & - & 1.000 \\
\hline
\end{tabular}

Note. - : Correlation is not identifiable.

Surrogacy analyses A sensitivity analysis was conducted where $R_{H}^{2}$ was estimated using 500 runs of the algorithm that was detailed in Section 4 . The grids $G=\{-1,-0.99999, \ldots, 1\}$ were used for all the unidentified correlations. A hierarchical approach with forward selection was used to identify the best set of surrogates. To this end, univariate analyses were conducted for each of the $p=67$ candidate surrogates (i.e., the OTUs at day 12). The candidate univariate surrogate that had the highest median $R_{H}^{2}$ was retained. In the second step, multivariate analyses were conducted that included the first identified candidate surrogate combined with a second one (i.e., one of the $p-1=66$ remaining OTUs). Again, for the 66 analyses conducted, the bivariate surrogate that led to the highest median $R_{H}^{2}$ was retained. This procedure was repeated until $\Delta S$ had a median $R_{H}^{2} \geq 0.90$. The so-obtained final vector of surrogates included $S_{1}=$ OTU $44, S_{2}=$ OTU 1 and $S_{3}=$ OTU 59 (OTU 44 belongs to the species Ruminococcaceae Ruminococcus, and both OTUs 1 and 59 belong to the species Verrucomicrobiaceae Akkermansia). The correlations between the true endpoint and these surrogates are shown in Table 1.

Table 2 and Figure 1 (top panel) show the main results for the analyses where $S_{1}-S_{3}$ are first considered in a univariate way, after which all possible pairs $\left(S_{1}, S_{2}\right),\left(S_{1}, S_{3}\right),\left(S_{2}, S_{3}\right)$ and the triplet $\left(S_{1}, S_{2}, S_{3}\right)$ were evaluated. In the univariate scenarios, it can be seen that 
$S_{1}$ was the best surrogate, with a relatively high median $R_{H}^{2}=0.754$. However, the range of the estimated $R_{H}^{2}$ values was very wide, with minimum $R_{H}^{2}=0.543$ (indicative of a poor surrogate) and maximum $R_{H}^{2}=0.956$ (indicative of an excellent surrogate). The range of the estimated $R_{H}^{2}$ values reflects the impact of the unverifiable assumptions on the results. The median $R_{H}^{2}$ for the univariate analyses that included $S_{2}$ and $S_{3}$ were substantially lower and equalled 0.280 and 0.145 , respectively. The ranges of the estimated $R_{H}^{2}$ values for these analyses were very large again and spanned nearly the entire unit interval. The adjusted association exhibited a similar pattern.

In the multivariate analyses, it can readily be observed that adding an additional surrogate to the existing one(s) always improves $R_{H}^{2}$ (in line with lemma 2). The best prediction of $\Delta T$ is thus obtained when all three surrogates are used in the analysis, with median $R_{H}^{2}=0.918$. As can be seen in Figure 1 (bottom panel), $R_{H}^{2}$ was high in all scenarios considered in the sensitivity analysis. Indeed, $R_{H}^{2}$ ranged between 0.751 and 0.999 , with $90 \%$ of the $R_{H}^{2}$ values exceeding 0.840 . Importantly, even though $S_{2}$ and $S_{3}$ had limited value as univariate surrogates (with median $R_{H}^{2} \leq 0.30$ ), the latter two surrogates led to a substantial increase in $R_{H}^{2}$ when considered jointly with $S_{1}$. This indicates that $S_{2}$ and $S_{3}$ contain useful information that is not captured by $S_{1}$, and it supports the claim that the use of multivariate surrogates can be a route to ameliorate the problems of finding a good surrogate. The estimated $\gamma_{\Delta}^{2}$ for the model that included all three surrogates was high as well and equalled $0.886(95 \% \mathrm{CI}=[0.765,1.000])$.

In all analyses, the bias-corrected $R_{H}^{2}$ and $\gamma_{\Delta}^{2}$ were slightly lower than the $R_{H}^{2}$ and $\gamma_{\Delta}^{2}$ (as expected), but the results were very similar and qualitatively the same. 


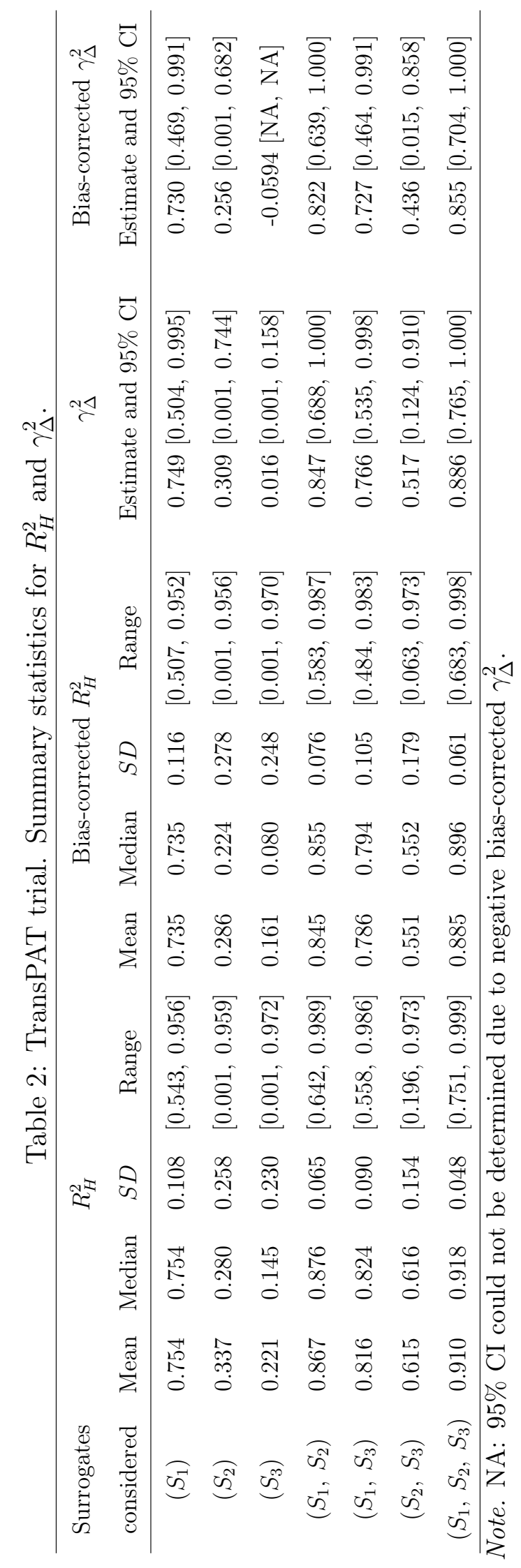



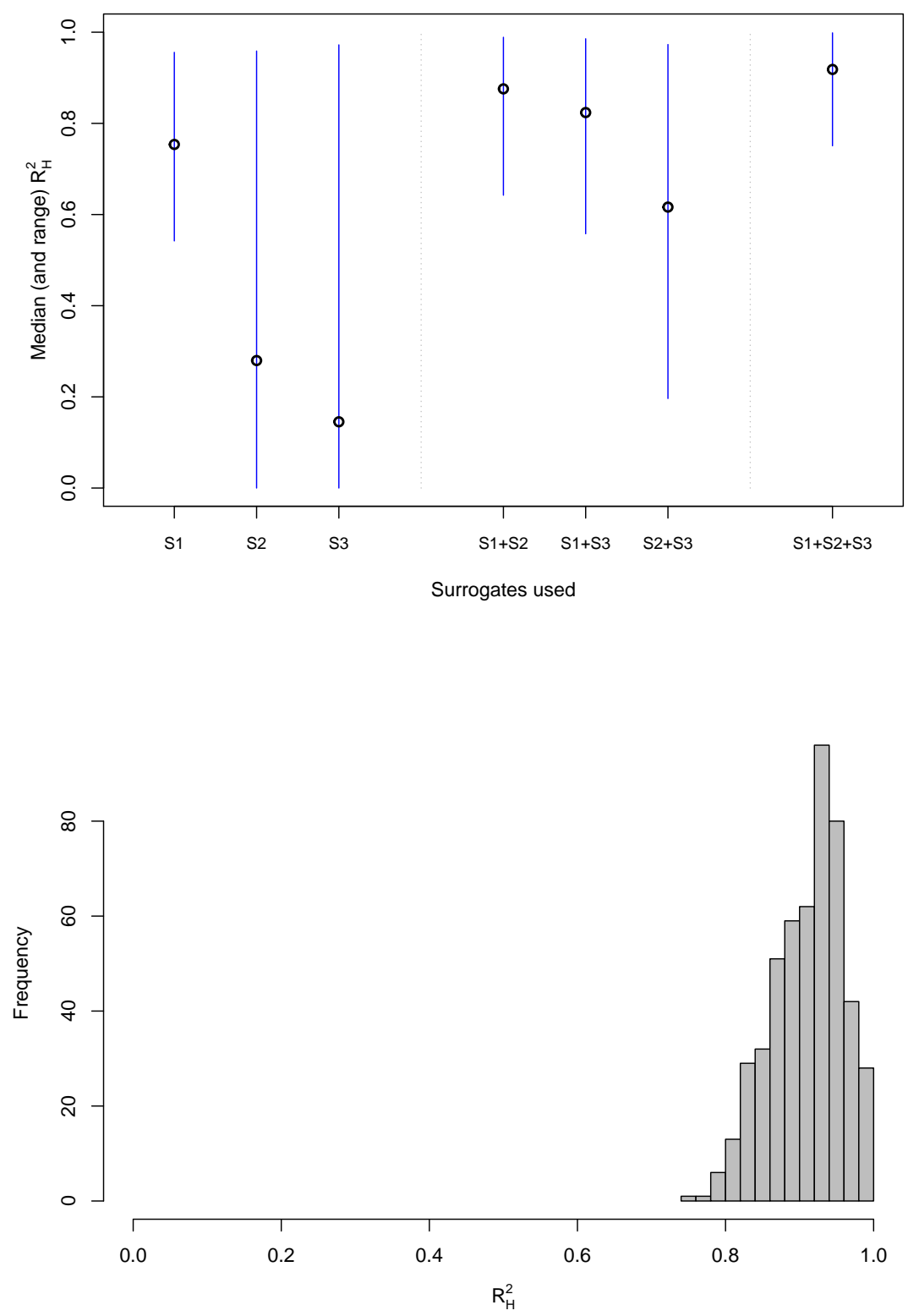

Figure 1: TransPAT study. Medians (circles) and ranges (solid vertical lines) of $R_{H}^{2}$ in the different surrogate evaluation scenarios (top figure). Histogram of $R_{H}^{2}$ when all three surrogates are used (bottom figure).

Setting biologically plausible constraints The grids $G=\{-1,-0.99999, \ldots, 1\}$ were used for all the unidentified correlations in the sensitivity analysis. In some situations, biological or subject-specific knowledge may impose some restrictions on the grid considered 
for one or more of the unidentifiable correlations. This may in turn lead to a narrower range of $R_{H}^{2}$ values. For example, suppose that experts consider it biologically implausible that $\mathrm{r}\left(S_{30}, S_{31}\right)>0$ and thus the grid $G=\{-1,-0.99999, \ldots, 0\}$ is used for the latter unidentified correlation in the sensitivity analysis. For the other unidentified correlations, no biological knowledge is available and the grid $G=\{-1,-0.99999, \ldots, 1\}$ is used. In the general analysis where $S_{1}, S_{2}$ and $S_{3}$ were used as surrogates, the range of $R_{H}^{2}$ was $[0.751,0.999]$. When applying the constraint that $\mathrm{r}\left(S_{30}, S_{31}\right) \leq 0$, the range (uncertainty) of $R_{H}^{2}$ slightly reduced to [0.789, 0.999]. Figure 3 graphically shows the relation between $\mathrm{r}\left(S_{30}, S_{31}\right)$ and $R_{H}^{2}$.

Web Appendix In the Web Appendix (Part II), it is exemplified how the surrogacy analyses can be conducted using the Surrogate R package.

\section{Simulation study}

Lemma 2 states that $R_{H}^{2}$ cannot decrease when additional surrogates are considered in the analysis. To further explore how $R_{H}^{2}$ changes when additional surrogates are considered, a small simulation study was conducted. To this end, a total of four surrogates $\left(S_{1}, S_{2}, S_{3}, S_{4}\right)$ were considered in four different scenarios where it was assumed that all identifiable correlations between $S_{k}$ and $T$ equalled $0,0.80,0.95$, or 0.99 , respectively (in both treatment conditions). $R_{H}^{2}$ was subsequently estimated using $S_{1}, S_{1}+S_{2}, S_{1}+S_{2}+S_{3}$,

and $S_{1}+S_{2}+S_{3}+S_{4}$. Of main interest is the reduction in the range of the $R_{H}^{2}$ values (i.e., uncertainty) when additional surrogates are considered. The analysis was conducted using the sensitivity-based approach that was described in Section 4 , with $M=500$ runs of the algorithm.

Results Figure 2 shows the histograms of the obtained $R_{H}^{2}$ in the different scenarios. The top panel in Figure 2 shows the results of the univariate analyses. In line with earlier simulation studies, $R_{H}^{2}$ is higher in scenarios where the correlation between $S$ and $T$ is higher (Van der Elst, Molenberghs and Alonso, 2016). However, in all univariate analyses, $R_{H}^{2}$ nearly spans the entire unit interval. Indeed, even when the correlation between $S$ 
and $T$ is assumed to be as high as $0.99, R_{H}^{2}$ ranges between 0.070 (indicative of a poor surrogate) and 0.999 (indicative of an excellent surrogate) and it can thus not conclusively be established that $\Delta S$ is a good surrogate for $\Delta T$.

Rows 2 to 4 in Figure 2 show the results of the multivariate analyses. As can be seen, the impact of adding extra surrogates on $R_{H}^{2}$ depends on the assumed identifiable correlations. In Scenario 1 (where all identifiable correlations are assumed to equal 0; independence), the consideration of additional surrogates does not lead to a reduction of the uncertainty in $R_{H}^{2}$. For example, even when all four surrogates are considered, $R_{H}^{2}$ still ranges between 0.005 and 0.862. In contrast, in Scenario 4 (where all identifiable correlations are assumed to equal 0.99 ), the range of $R_{H}^{2}$ decreases substantially when more surrogates are considered. For example, when four surrogates are used in the analysis, $R_{H}^{2}$ ranges between 0.948 and 0.999 and thus the qualitative conclusion that $\Delta \boldsymbol{S}$ is an excellent surrogate for $\Delta T$ is the same in all realities compatible with the identifiable correlations. The same pattern of results can be observed in Scenarios 2 and 3 (where all identifiable correlations are assumed to equal 0.50 and 0.95 , respectively), but the extent to which the uncertainty in $R_{H}^{2}$ is reduced is smaller compared to what is the case in Scenario 4 .

Conclusion The results of the simulation study indicate that the addition of extra surrogates leads to a reduction of the uncertainty in $R_{H}^{2}$, with larger reductions occurring when the identifiable correlations in the data are higher. For putative surrogates that are independent from $T$, the reduction in the uncertainty is negligible. Further, it is noteworthy that in some scenarios (especially when multiple 'good' surrogates are considered) the range of the obtained $R_{H}^{2}$ values becomes small and the unidentifiability problem is no longer an issue from a practical perspective as the qualitative conclusion of the analysis is the same in all 'plausible realities' compatible with the identifiable correlations. In the Web Appendix Part III, an additional simulation study is described that leads to essentially the same conclusions.' 

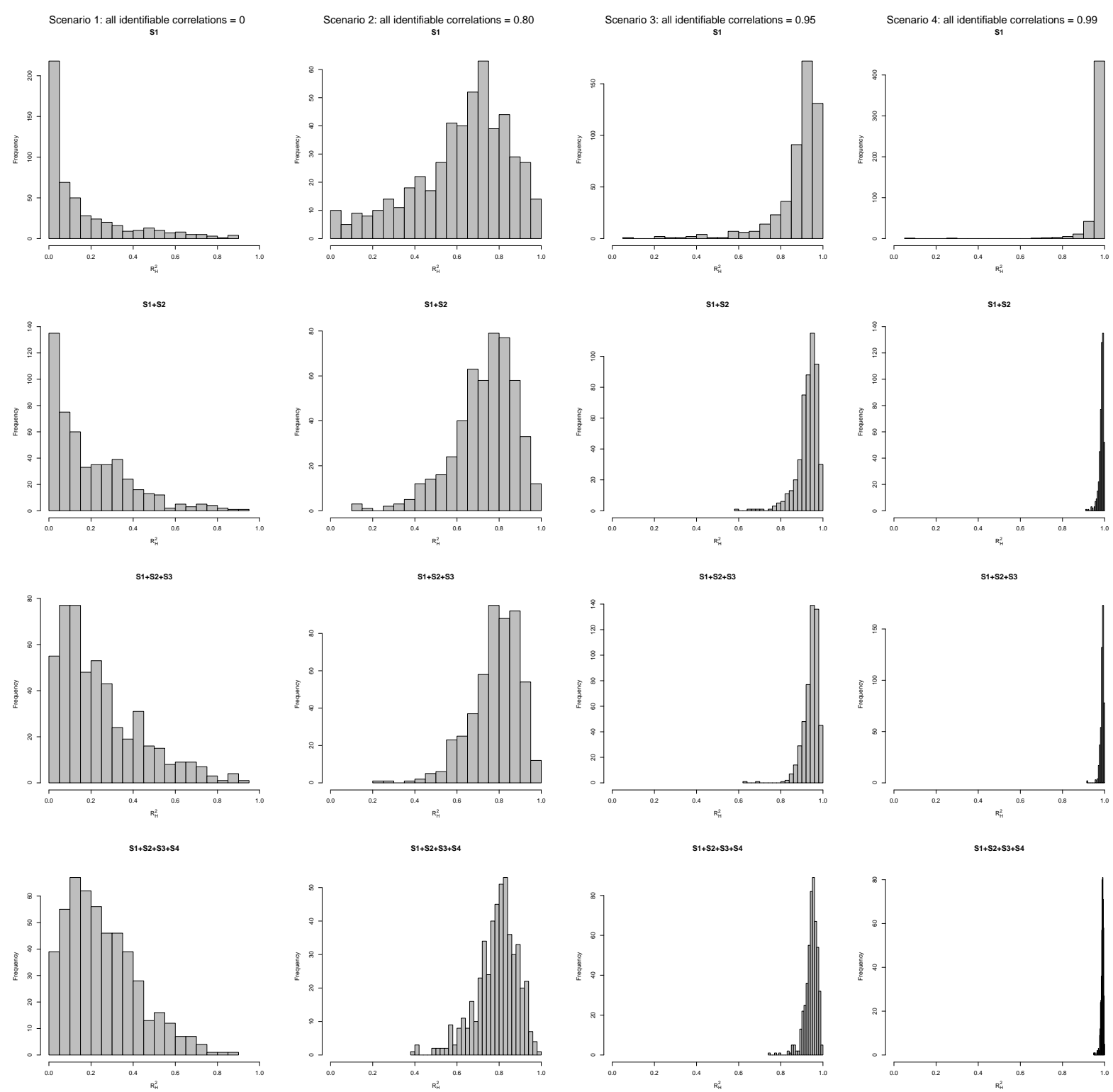

Figure 2: Results of the simulation study. Histograms of $R_{H}^{2}$ as a function of the number of surrogates used in the scenarios where all identifiable correlations equal 0 (1st column), 0.80 (2nd column), 0.95 (3rd column), and 0.99 (4th column). 


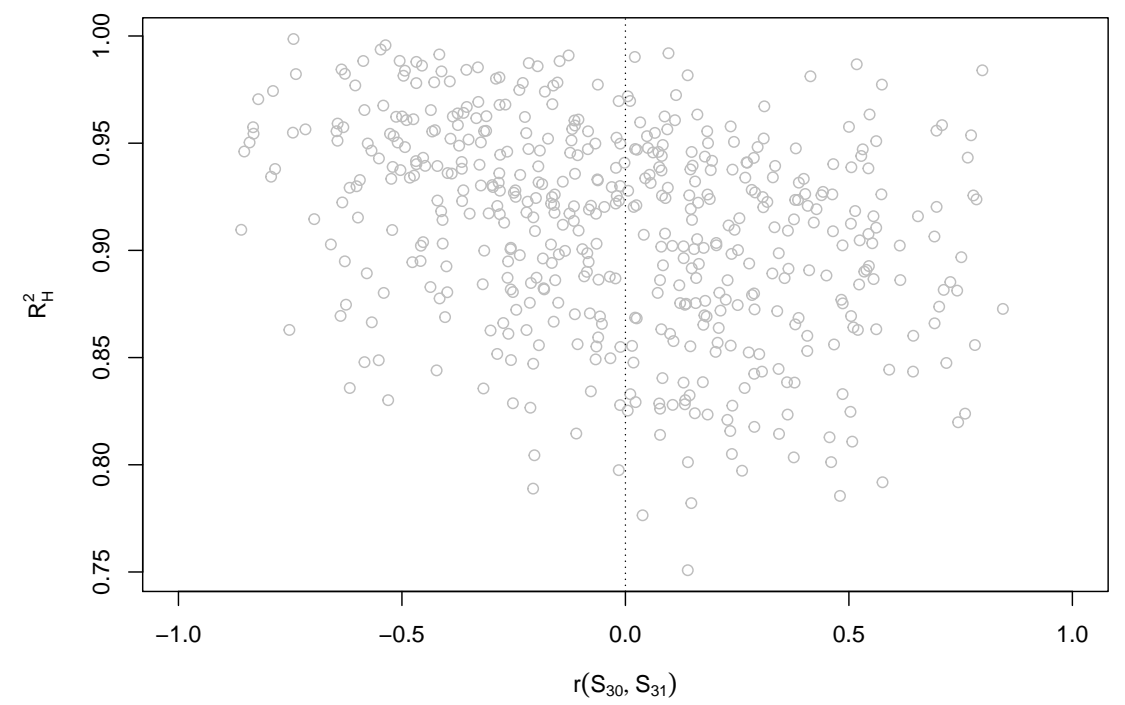

Figure 3: TransPAT trial. Relation between $\mathrm{r}\left(S_{30}, S_{31}\right)$ and $R_{H}^{2}$ in the analysis where $S_{1}, S_{2}$ and $S_{3}$ were used as surrogates in the 500 runs of the sensitivity analysis. Observations for which $\mathrm{r}\left(S_{30}, S_{31}\right) \leq 0$ (indicated by a vertical dashed line) are considered more biologically plausible.

\section{Discussion}

At present, most surrogate evaluation methods allow for considering only one surrogate endpoint. Given the complex nature of many diseases and the various therapeutic pathways in which a treatment may impact the clinical outcome, it seems reasonable to expect that the prediction of the treatment effect on the true endpoint can be substantially improved when multiple surrogates are considered rather than only a single one. An example of this was provided in the case study.

Two new metrics of surrogacy were proposed, i.e., the multivariate ICA $\left(R_{H}^{2}\right)$ and the multivariate adjusted association $\left(\gamma_{\Delta}^{2}\right) . \quad R_{H}^{2}$ and $\gamma_{\Delta}^{2}$ are complementary metrics of surrogacy that have a different interpretation. The former quantifies the association between the individual causal treatment effects on the surrogates and the true endpoint, whereas the latter quantifies the treatment-corrected correlation between the surrogates and the true endpoint. Nowadays, there is wide consensus that a correlate does not make a surrogate (Alonso et al., 2016), but a strong correlation between $\boldsymbol{S}$ and $T$ (i.e., a high $\gamma_{\Delta}^{2}$ ) is nonetheless an intuitively desirable condition for surrogacy. In the univariate setting, Alonso et al. 
(2015) stated that the stronger the correlation between the putative $S$ and $T$ is, the more probable it is that the former will be a valid surrogate endpoint. This is even more so in the multivariate scenario. Indeed, the simulation study discussed in Section 7 showed that $R_{H}^{2}$ spans nearly the entire unit interval in the univariate setting, even when the correlation between $S$ and $T$ is as high as 0.99. A (univariate) correlate thus does not make a surrogate, in the sense that a high correlation between $S$ and $T$ does not guarantee a high $R_{H}^{2}$. However, when a sufficiently large set of surrogates that are all highly correlated with $T$ is considered, all $R_{H}^{2}$ are close to 1 . The simulation study thus suggests that the claim that a correlate does not make a surrogate can be relaxed in the multivariate setting, but more extensive simulations are needed to further corroborate this claim.

A Monte Carlo procedure was proposed to address the identifiability problems that occur in the estimation of $R_{H}^{2}$. It is important to point out that this Monte Carlo procedure is not designed to estimate the unidentifiable $R_{H}^{2}$, but rather to offer a sensitivity analysis. Basically, the vector of $R_{H}^{2}$ values that is obtained from the procedure assess the ICA across 'plausible realities', i.e., across realities that are compatible with the data at hand. Similar ideas were proposed by Molenberghs et al. (2015), who used the 'region of ignorance' concept (which contains estimators that are consistent with the observed data) in a missing data context.

Some critical comments and suggestions for future research can be given. First, the algorithm to identify $\Sigma$ and compute $R_{H}^{2}$ allows for including an unlimited number of candidate surrogates. However, from a practical (i.e., computational) perspective, the current implementation of the algorithm is mainly suitable to consider at most 3 or 4 surrogates. For example, in the case study it took on average about $0.5 \mathrm{sec} ., 15 \mathrm{sec}$, and 2 hours to find $M=500$ valid $\boldsymbol{\Sigma}$ matrices in the analyses where 1, 2 and 3 surrogates were used, respectively. The exponential increase in computation time is directly related to the problem of generating large positive definite matrices that are subject to certain constraints (i.e., the identifiable correlations). This problem is known as the 'matrix completion problem' in the literature. Even though in most practical settings the use of 3 to 4 surrogates may be sufficient, it would be worthwhile to further explore alternative algorithms (i) that allow for an increase in the dimension of $\Sigma$ for which valid solutions can be found, and (ii) that 
increase the speed by which valid solutions for $\Sigma$ are found. This is a topic of ongoing research (Flórez et al., 2018).

Second, in the analysis of the case study, the identifiable covariances and correlations were fixed at their estimated values in the computation of $R_{H}^{2}$. To account for the sampling variability in the estimates of the identifiable parameters, one could specify distributions for the identifiable parameters rather than fixing them at their point estimate. For example, uniform distributions could be specified for the identifiable correlations with [min, $\max$ ] values equal to their estimated upper and lower 95\% confidence interval bounds. Then random values can be drawn from the specified distributions in each run of the algorithm to compute $R_{H}^{2}$ (for an example in the univariate setting, see Van der Elst, Molenberghs and Alonso, 2016).

Third, there is always an increase in $R_{H}^{2}$ and $\gamma_{\Delta}^{2}$ when additional surrogates are added to the existing ones. The question may arise how large this increase should be in order to retain the additional candidate surrogate endpoint(s). In most clinical trials, adding additional surrogates will increase the burden to the patients and/or the research nurses, the financial cost of the clinical trial, and/or the probability of having missing values. Experts in the field should carefully balance the 'costs' of considering additional surrogates against their 'benefits' in terms of increased prediction accuracy (e.g., the increase in the median $R_{H}^{2}$ values). Assam et al. (2010) formalized such an approach in a meta-analytic surrogate evaluation context where a longitudinal sequence of surrogates was considered. These authors specified an objective function that contains a prediction accuracy component, namely, the increase in individual-level surrogacy and a cost component, i.e., the financial cost per additional repeated measurement of the surrogate. The objective function is subsequently maximized to determine the optimal number of measurements, where the importance of the prediction accuracy and the cost components can be gauged through the use of weights. Similar ideas could be used in the multivariate surrogacy setting that was considered in the present paper, but this goes beyond the scope of the present work and is a topic for future research. 


\section{Supplementary Materials}

The methodology that is proposed in this manuscript is implemented in the R package Surrogate (available for download at https://CRAN.R-project.org/package=Surrogate). A Web Appendix that contains the proofs of Lemma's 1 and 2 (Part I of the Web Appendix), that details the analysis of the case study using the R package Surrogate (Part II), and that provides a second simulation study to evaluate the impact of adding additional surrogates on $R_{H}^{2}$ (Part III) is available. 


\section{References}

Alonso, A. A., Van der Elst, W., Molenberghs, G., Buyse, M., and Burzykowski, T. (2015). On the relationship between the causal-inference and meta-analytic paradigms for the validation of surrogate endpoints. Biometrics 71, 15-24.

Alonso, A. A., Bigirumurame, T., Burzykowski, T., Buyse, M., Molenberghs, G., Muchene, L., Perualila, N. J., Shkedy, Z., and Van der Elst, W. (2016). Applied surrogate endpoint evaluation methods with SAS and R. New York: CRC Press.

Assam N. P., Tilahun, E. A., Alonso A. A., and Molenberghs, G. (2010). Using earlier measures in a longitudinal sequence as a potential surrogate for a later one. Computational Statistics 85 Data Analysis, 54, 1342-1354

Burzykowski, T., Molenberghs, G., and Buyse, M. (2005). The Evaluation of Surrogate Endpoints. New York: Springer-Verlag.

Buyse, M., and Molenberghs, G. (1998) The validation of surrogate endpoints in randomized experiments. Biometrics 54, 1014-1029.

Buyse, M., Molenberghs, G., Burzykowski, T., Renard, D., and Geys, H. (2000). The validation of surrogate endpoints in meta-analyses of randomized experiments. Biostatistics $1,49-67$.

Buyse, M., Sargent, D. J., Grothey, A., Matheson, A. and de Gramont, A. (2010). Biomarkers and surrogate end points-the challenge of statistical validation. Nat. Rev. Clin. Oncol. 7, 309-317.

Cohen, J., Cohen, P., West, S. G., and Aiken, L. S. (2003). Applied multiple regression/correlation analysis for the behavioral sciences. Third edition. New Jersey: Lawrence Erlbaum Associates.

Conlon, A. S. C., Taylor, J. M. G., and Elliott, M. R. (2013). Surrogacy assessment using principal stratification when surrogate and outcome measures are multivariate normal. Biostatistics 14, 1-18. 
Elliott, M.R., Li, Y., and Taylor, J.M.G. (2013). Accommodating missingness when assessing surrogacy via principal stratification. Clinical Trials 10, 363-377.

Fitzmaurice, G., Davidian, M., Verbeke, G., and Molenberghs, G. (2009). Longitudinal data analysis. New York: CRC press.

Fleming T. R. and DeMets D. L. (1996). Surrogate endpoints in clinical trials: are we being misled? Annals of Internal Medicine 125, 605-613.

Flórez A. J., Alonso A. A., Molenberghs G. and Van der Elst W. (2018). Generating correlation matrices with fiixed values: an application to the evaluation of multivariate surrogate endpoints.

Freedman L. S., Graubard, B. I., and Schatzkin, A. (1992). Statistical validation of intermediate endpoints for chronic diseases. Statistics in Medicine 11, 167-178.

Holland, P. W. (1986). Statistics and Causal Inference. Journal of the American Statistical Association 8, 945-960.

Joe, H. (1989). Relative Entropy Measures of Multivariate Dependence. Journal of the American Statistical Association 84, 157 - 164.

Linfoot E. H. (1957). An informational measure of correlation. Information and Control 1, $85-89$.

Molenberghs, G., Fitzmaurice, G., Kenward, M. G., Tsiatis, A., and Verbeke, G. (2015). Handbook of missing data methodology. New York: CRC Press.

Prentice, R. L. (1989). Surrogate endpoints in clinical trials: definitions and operational criteria. Statistics in Medicine 8, 431-440.

Rousseeuw, P. J., and Molenberghs, G. (1994). The shape of correlation matrices. The American Statistician 48, 276-279.

Rosenbaum, P. R. and Rubin, D. B. (1983). The central role of the propensity score in observational studies for causal effects. Biometrika 70, 41-55. 
Rubin, D. B. (1986). Statistics and causal inference: Comment: which ifs have causal answers. Journal of the American Statistical Association 81, 961-962.

Ruiz, V. E. et al. (2017). A single early-in-life macrolide course has lasting effects on murine microbial network topology and immunity. Nature Communications 8, 1-14.

Van der Elst, W., Molenberghs, G., \& Alonso, A. A. (2016). Exploring the relationship between the causal-inference and meta-analytic paradigms for the evaluation of surrogate endpoints. Statistics in Medicine 35, 1281-1298.

Yin, P., and Fan, X. (2001). Estimating $R^{2}$ shrinkage in multiple regression: A comparison of different analytical methods. The Journal of Experimental Education 69, 203-224. 